\title{
Revisión por pares: evidencias y desafíos
}

\section{Peer review: evidence and challenges}

\author{
Luisa Schonhaut Berman ${ }^{\mathrm{a}, \mathrm{b}}$, Teresa Millán Klusse ${ }^{\mathrm{a}, \mathrm{c}}$, Loreto Podestá López ${ }^{\mathrm{a}, \mathrm{d}}$ \\ aComité editorial, Revista Chilena de Pediatría \\ ${ }^{b}$ Clínica Alemana de Santiago, Facultad de Medicina, Universidad del Desarrollo, Santiago, Chile \\ 'Departamento de Pediatría y Cirugía Infantil Occidente, Facultad de Medicina, Universidad de Chile, Santiago, Chile \\ dInstituto de Pediatría, Facultad de Medicina, Universidad Austral de Chile, Valdivia, Región de los Ríos, Chile
}

Visibilidad y factor de impacto de las publicaciones científicas

La investigación clínica, epidemiológica y tecnológica nos ha llevado al avance científico, contribuyendo al desarrollo y expansión del conocimiento en forma continua e incremental. El proceso de investigación finaliza una vez que los resultados son publicados, y desde muy antiguo en la comunidad científica se promueve la máxima de que sólo cuando es publicada, la ciencia existe.

La necesidad de sustentar la investigación local y dar un espacio para su difusión, motivó a distintas instituciones y sociedades científicas a tener su propia revista, la que, en la medida que crece y va adquiriendo prestigio, recibe artículos desde otras instituciones locales y distintas latitudes del planeta, ganando así visibilidad global e impacto científico.

El factor de impacto es una herramienta de evaluación cienciométrica y bibliométrica que sirve para comparar revistas científicas y evaluar la importancia relativa de cada una según las citas recibidas por los artículos que publica. El índice empleado con mayor frecuencia es el "Journal Impact Factor" (JIF) que es generado anualmente por el Journal of Citation Reports del Institute of Scientific Information (ISI), actualmente Thomson Reuters.
Por otra parte el SCImago Research Group, grupo de investigación que emplea datos de Scopus, una base de datos que además de entregar información bibliográfica publica índices para medir la calidad de las revistas científicas, calcula el indicador SCImago Journal Rank (SJR), que agrega una ponderación adicional al área de investigación, la calidad y reputación de la revista, considerando que estos factores impactan e influyen en el valor de la citación. Clasifica además las publicaciones en cuartiles, comparando la posición de una revista en relación con todas las de su área, de modo que las revistas con el factor de impacto más alto estarán en el primer cuartil.

El factor de impacto puede aumentar mientras mayor sea el número de veces que los artículos son citados y/o al disminuir el número de artículos publicados, dejando fuera los que tienen menor probabilidad de ser referenciados, ya sea por su calidad técnica u originalidad

La Revista Chilena de Pediatría (RCHP), publicación oficial y autónoma de la Sociedad Chilena de Pediatría cumplió recientemente 87 años de publicación bimestral ininterrumpida. Desde su ingreso el año 1998 a la red Scientific ELectronic Library On Line (SciELO), una de las primeras estrategias open Access para la difusión de las publicaciones, ha logrado cada vez mayor visibilidad e impacto dentro de las publica- 
ciones internacionales hispanoparlantes, lo que significó que el 2014 fuese indexada en la base de datos de la National Library of Medicine, Medline, y su motor de búsqueda PubMed, y, que en el año 2016 se clasificara en el tercer cuartil de publicaciones pediátricas internacionales (SJR). A partir del 2017 comenzó a publicarse en forma bilingüe, en idiomas español e inglés, con lo que se espera aumente aún más su impacto en la comunidad científica.

En este camino de continuo crecimiento y consolidación editorial, es de la mayor importancia contar con el respaldo y compromiso de una institución matriz. RCHP ha nacido y crecido al alero de la Sociedad Chilena de Pediatría, junto con el aporte de los autores que envían sus manuscritos, la labor de editores, y especialmente de los revisores, quienes son eslabon fundamental en el proceso editorial a través de su participación en la "revisión por pares" (RPP).

\section{La revisión por pares o "peer review"}

La RPP o "peer review" ha sido una parte formal de la comunicación científica desde la aparición de las primeras revistas hace más de 300 años, siendo la revista Philosophical Transactions de la Royal Society pionera en este proceso $^{1}$. Si bien la RPP ha enfrentado controversias debido a que enlentece el proceso editorial, puede fallar en la detección de errores, además de estar sujeta a sesgos y malas prácticas éticas; aunque falta evidencia empírica que demuestre su validez ${ }^{2,3} \sin$ duda garantiza la transparencia, credibilidad y prestigio de las publicaciones ${ }^{4,5}$.

Se habla de RPP cuando un revisor o árbitro experto, externo a la revista, independiente y libre de conflicto de interés realiza la evaluación de un manuscrito, en un plazo estipulado. En su evaluación crítica el revisor debe valorar el artículo en cuanto a su aporte al conocimiento, calidad, diseño, metodología, estructura, originalidad, validez de la discusión y conclusiones y, también, en la posibilidad de fraude. Además de sus comentarios, el revisor aporta recomendaciones al autor que permiten mejorar el manuscrito y aconseja al editor acerca de si este puede ser publicado, si requiere modificaciones o si no debería publicarse. ${ }^{6}$ Si bien el revisor ayuda a seleccionar los mejores artículos, es el editor quien toma la decisión final acerca de si es publicable ${ }^{7}$.

La RCHP utiliza la modalidad de RPP denominada doble ciego, debido a que los autores desconocen la identidad de los revisores y viceversa. Existen otros tipos de RPP como la de simple ciego, en que sólo los autores desconocen la identidad de los revisores. Se han reportado experiencias con revisiones abiertas, que son las más transparentes, en que el revisor "firma" su ar- bitraje y sus créditos aparecen en el manuscrito junto con la revisión. En la era de las telecomunicaciones ha empezado a emplearse la alternativa de someter los "pre-print", es decir, los artículos previos a su publicación, a una revisión abierta en que toda la comunidad científica está invitada a exponer sus críticas; también se ha reportado experiencias con "post-print", en que los artículos ya publicados son comentados por los lectores. Aún hace falta mucha investigación para evaluar los pros y contras de cada uno de estos tipos de revisión ${ }^{8}$.

Cualquiera sea la modalidad de RPP utilizada, siempre existe posibilidad de malas prácticas en el proceso de revisión, por lo que es fundamental que todos los involucrados se comporten de manera responsable y ética. El Committee on Publication Ethics (COPE) publica guías de ética editorial para los editores y revisores, destacando que es reponsabilidad de los revisores, previo a aceptar la solicitud de arbitraje, chequear que tienen la experticia y tiempo necesario para completarla en el tiempo solicitado, en ausencia de conflicto de intereses?.

Contar con revisiones oportunas y de calidad es una piedra angular en los procesos editoriales, en que existe una presión cada vez mayor por acortar los tiempos de revisión, edición y publicación. No obstante, esta demanda es contrarrestada con las posibilidades de los revisores, cuya labor demanda tiempo y dedicación, sin ser remunerada y pocas veces reconocida académicamente.

El editor selecciona un revisor en base a la temática del manuscrito y metodología estadística aplicada, considerando además su historia de cumplimiento y calidad de revisiones. Se ha reportado que hay una mayor tasa de respuestas cuando el revisor es conocido por el editor, por lo que es importante contar con un comité editorial diverso con redes y contactos. Tal ha sido la política de la RCHP, que a lo largo de los últimos años ha ampliado la representatividad del comité editorial, con participación de investigadores de distintas instituciones científicas tanto de Santiago como de regiones.

El trabajo de los revisores puede ser ponderado a partir de la calidad de la revisión, el aporte constructivo a la mejora del manuscrito y cumplimiento en el tiempo solicitado. Matías-Guiu y cols. ${ }^{10}$ analizaron el perfil de los evaluadores de la Revista de Neurología según tasa de respuesta, encontrando que los mejores respondedores son los árbitros de menor edad y de sexo femenino, sin demostrarse un efecto fatiga en los buenos árbitros. Kliewer y cols. demostraron que la calidad de los revisores depende fundamentalmente de factores personales, siendo superiores las revisiones hechas por profesionales más jóvenes pertenecientes a instituciones académicas ${ }^{10}$. Cuando la invitación a re- 
Tabla 1 . Lista de chequeo para los autores, previo al envío del manuscrito*

\begin{tabular}{|c|c|c|c|}
\hline \multirow[t]{4}{*}{ Aspectos éticos } & ¿Tiene el consentimiento/asentimiento informado? & sí( ) & no () \\
\hline & ¿Tiene aprobación del comité de ética? & sí( ) & no () \\
\hline & ¿Su artículo es original? ¿No contiene elementos plagiados o autoplagiados? & sí( ) & no () \\
\hline & ¿Su artículo no ha sido enviado simultáneamente a otra publicación? & sí( ) & no () \\
\hline Título & ¿Refleja en forma clara los contenidos del estudio? & sí ( ) & no () \\
\hline \multirow[t]{4}{*}{ Resumen } & ¿Está estructurado de acuerdo a formato IMRyD incluyendo los objetivos? & sí ( ) & no () \\
\hline & ¿El cuerpo del resumen deja claro el contenido del manuscrito (metodología/caso clínico)? & sí( ) & no () \\
\hline & ¿Tiene un máximo de 250 palabras? & sí( ) & no ( ) \\
\hline & ¿Adjuntó el resumen en inglés? & sí ( ) & no () \\
\hline \multirow[t]{2}{*}{ Palabras claves } & ¿Tiene al menos 3 palabras claves? & sí ( ) & no () \\
\hline & ¿Son palabras Mesh? & sí ( ) & no () \\
\hline \multirow[t]{4}{*}{ Introducción } & ¿Permite contextualizar el trabajo? & sí( ) & no () \\
\hline & ¿Están los objetivos al final de la introducción? & sí( ) & no () \\
\hline & ¿Las referencias son actualizadas y suficientes? & sí ( ) & no ( ) \\
\hline & ¿Tiene una extensión similar a la discusión? & sí ( ) & no () \\
\hline \multirow[t]{6}{*}{ Pacientes y método } & ¿Se describe el diseño del estudio? & sí ( ) & no () \\
\hline & ¿La metodología es apropiada? ¿Está bien descrita? & sí ( ) & no ( ) \\
\hline & ¿La muestra es suficiente para obtener conclusiones? & sí ( ) & no ( ) \\
\hline & Si su estudio es cuantitativo ¿Describe las estadísticas empleadas y programa estadístico? & sí( ) & no () \\
\hline & ¿Está redactado en tiempo pasado? & sí ( ) & no () \\
\hline & ¿Revisó las guías EQUATOR correspondientes al diseño de su estudio? & sí ( ) & no () \\
\hline \multirow[t]{4}{*}{ Resultados } & ¿Son claros? & sí ( ) & no () \\
\hline & ¿Son creíbles? & sí( ) & no () \\
\hline & ¿Están bien descritos? ¿Tienen una secuencia lógica? & sí( ) & no () \\
\hline & ¿Describen solamente los hallazgos más importantes sin repetir las tablas y/o figuras? & sí ( ) & no ( ) \\
\hline \multirow[t]{2}{*}{ Caso clínico } & ¿Está redactado en tiempo pasado? & sí ( ) & no () \\
\hline & ¿Revisó las guías CARE para reporte de caso? & sí ( ) & no ( ) \\
\hline \multirow[t]{6}{*}{ Tablas y figuras } & ¿Ayudan? & sí ( ) & no ( ) \\
\hline & ¿Son claras? ¿Son autoexplicativas? & sí ( ) & no ( ) \\
\hline & ¿Los datos son concordantes con los resultados del manuscrito? & sí ( ) & no ( ) \\
\hline & En caso de abreviaturas ¿Son universales, y/o están explicitadas al pie de tabla/figura? & sí ( ) & no ( ) \\
\hline & ¿Están enumeradas en el texto? & sí ( ) & no ( ) \\
\hline & ¿No excede más de 5 tablas y/o figuras en total? & sí ( ) & no ( ) \\
\hline \multirow[t]{5}{*}{ Discusión } & ¿Comienza con los resultados más relevantes? & sí ( ) & no ( ) \\
\hline & ¿Confronta los resultados con la literatura publicada? & sí ( ) & no ( ) \\
\hline & ¿Se describen las limitaciones del estudio? & sí ( ) & no ( ) \\
\hline & ¿Se describen las fortalezas del estudio y su aporte al conocimiento científico? & sí ( ) & no ( ) \\
\hline & ¿Tiene apoyo bibliográfico suficiente y actualizado? & sí ( ) & no ( ) \\
\hline Conclusiones & ¿Son concordantes con los objetivos y resultados? & sí ( ) & no ( ) \\
\hline \multirow[t]{5}{*}{ Referencias } & ¿Son suficientes? Si es artículo original máximo 40, caso clínico entre 7 y 25. & sí ( ) & no ( ) \\
\hline & ¿Son correctas? & sí ( ) & no ( ) \\
\hline & ¿Le parece que falta alguna referencia importante? & sí ( ) & no ( ) \\
\hline & ¿Están en formato VANCOUVER? & sí ( ) & no ( ) \\
\hline & ¿No supera el $15 \%$ de auto-referencias de artículos publicados en la misma revista? & sí ( ) & no ( ) \\
\hline \multirow[t]{2}{*}{ Originalidad } & ¿El artículo aporta algo nuevo? & sí ( ) & no ( ) \\
\hline & ¿Abre nuevas preguntas de investigación? & sí ( ) & no () \\
\hline Relevancia & ¿La temática es apropiada a la línea editorial de la revista? & sí ( ) & no ( ) \\
\hline Extensión & $\begin{array}{l}\text { Si es un artículo original: ¿No supera las } 3500 \text { palabras? } \\
\text { Si es caso clínico: ¿ No supera } 2000 \text { palabras excluyendo la página de títulos y resumen? }\end{array}$ & sí ( ) & no ( ) \\
\hline
\end{tabular}

*En caso de tener alguna respuesta negativa, revise y corrija su manuscrito.

Referencias: Prince E. $2001^{13}$ y González de Dios J y cols. 2014 ${ }^{14}$.

IMRyD: I = introducción; $M=$ material y métodos; $R=$ resultados; y =y, D = discusión. En Caso Clínico: Introducción, caso clínico, conclusiones . 
visar un artículo proviene de una revista de prestigio, suele ser considerada un honor, lo que no ocurre con las revistas más pequeñas, que se ven enfrentadas a mayores dificultades para encontrar revisores disponibles. Es por ello que el grupo de editores de las revistas de las sociedades de pediatría del Cono Sur ha desarrollado como proyecto colaborativo una plataforma digital común de árbitros. ${ }^{11}$

En la RCHP, anualmente se extienden más de 500 solicitudes para hacer revisión de artículos, con una tasa de respuesta cercana al 50\%. En los últimos 2 años han colaborado más de 150 revisores/año, completando un promedio cercano a 2 revisiones anuales por árbitro. Se han buscado distintas estrategias para reconocer a los revisores, por su dedicación y trabajo, como publicar sus nombres en el último número de la revista, o premiar a los más destacados. Otras alternativas han sido otorgar créditos como parte de la formación médica continua, posibilitar el acceso abierto a bibliotecas de revistas o invitarlos a escribir una editorial relacionada. Se ha discutido incluso si debería haber un pago por revisión, asumiendo que algunas revistas han comenzado a cobrar por publicar, lo que permite sustentabilidad de las publicaciones. La RCHP difunde el listado de revisores que participaron durante el año calendario en el último fascículo de cada año, $y$, a partir del 2016 se ha presentado un cuadro de honor con los revisores más destacados en el Congreso Chileno de Pediatría.

\section{¿Es posible que el proceso de revisión por pares sea objetivo?}

Sin duda el proceso de revisión es subjetivo, por lo que se han desarrollado guías de apoyo para facilitar y hacer más objetivas las revisiones. En la tabla 1 adjuntamos una propuestas de lista de chequeo para los autores previo al envío de un manuscrito y en la tabla 2 la propuesta de lista de chequeo para los revisores, ambas basadas en guías publicadas y aceptadas internacionalmente ${ }^{12-14}$. Además se han desarrollado guías específicas para los distintos diseños de investigación, que están disponibles en EQUATOR (Enhancing the QUAlity and Transparency Of health Research) $h t t p: / /$ www.equator-network.org. Estas guías ayudan tanto a revisores como a los autores a identificar los elementos mínimos de calidad de una publicación. En un ensayo randomizado y controlado, Cobo y cols. demostraron que la revisión basada en estas guías, comparada con la

\section{Tabla 2. Lista de chequeo para los revisores}

\begin{tabular}{ll}
\hline Antes de empezar la revisión* & Verifique que tiene la experticia suficiente en el tema del artículo \\
& Verifique que no tiene conflicto de intereses \\
& Asegúrese de tener el tiempo necesario para completar la revisión en el plazo solicitado \\
& Comience su revisión con un breve resumen del trabajo \\
Estructure su revisión & Estructure sus comentarios desde las observaciones mayores a las menores \\
& Enumere sus comentarios \\
Reglas generales de los buenos & Haga comentarios específicos y constructivos \\
revisores & Más que dar su opinión, entregue fundamentos objetivos \\
& No trate de cambiar el manuscrito, haga sugerencias para mejorarlo \\
& No ofenda a los autores \\
& Privilegie las críticas constructivas \\
& Evite mencionar su posición respecto a la publicabilidad del manuscrito en los comentarios a los autores \\
& ¿Es relevante la pregunta de investigación? \\
& La pregunta de investigación: ¿ Es original? ¿Aporta nueva información? \\
& La metodología: ¿Corresponde a la pregunta de investigación? \\
& Elija las guía EQUATOR de revisión apropiadas al diseño del manuscrito \\
¿Se presentan bien los resultados? & ¿Hay información redundante? ¿Faltan datos/referencias importantes? \\
revisado los siguientes aspectos & ¿La discusión está bien orientada? ¿Plantea nuevas interrogantes? \\
¿Están detalladas las limitaciones del estudio en la discusión? & ¿Las conclusiones están basadas en los resultados?
\end{tabular}

*En caso de no tener la experticia suficiente, el tiempo o si tiene conflicto de intereses por favor, excúsese de la revisión y sugiera algún revisor alternativo si conoce.

Referencia: Spigt M y Arts ICW. 201012. 
RPP convensional, mejoraba la calidad de los manuscritos $^{15}$. Entre estas guías encontramos las CONSORT para la evaluación de ensayos clínicos controlados; STROBE para estudios observacionales; PRISMA para revisiones sistemáticas; SRQR para estudios cualitativos; STARD para estudios de diagnóstico; ; CARE para casos clínicos; SQUIRE para estudios de calidad y mejora continua; CHEERS para evaluaciones económicas y SPIRIT para evaluar protocolos de investigación. La mayoría de estas guías están disponibles en español. http://www.equator-network.org/library/translationsof-reporting-guidelines/\#Spanish.

\section{Conclusiones}

Si bien la RPP no está libre de controversias, sin duda resguarda la calidad y apoya la credibilidad de las publicaciones científicas a través de la mirada crítica de los revisores, su asesoría al cuerpo editorial respecto a si los manuscritos deben ser publicados y sobre todo, por su aporte a la mejora de los mismos. Las guías basadas en la evidencia ayudan a los revisores a orientar, estandarizar y hacer más objetivo el proceso de RPP, por lo que invitamos a nuestros autores a examinarlas cuando redactan el manuscrito y a los revisores a utilizarlas en sus arbitrajes.

\section{Referencias}

1. Spier R. The history of the peer-review process. Trends Biotechnol. 2002;20: 357-8.

2. Jefferson T, Rudin M, Brodney Folse S, et al. Editorial peer review for improving the quality of reports of biomedical studies. Cochrane Database Syst Rev 2007;(2):MR000016.

3. Walker R and Rocha da Silva P. Emerging trends in peer review-a survey. Front Neurosci. 2015;9:169.

4. Alfonso F. Una revisión crítica del proceso de "peer review". Arch. Cardiol. Méx. 2010;80(4):272-82.

5. Mahawar KK, Kejariwal D, Malviya A, Birla R, Viswanath YKS. Peer Review Practices in Biomedical Literature: A Time for Change? Asian J Surg. 2009;32(4):2406.

6. Matías-Guíu J, García-Ramos R. Fraude y conductas inapropiadas en las publicaciones científicas. Neurología. 2010;25: 1-4.

7. Editorial Policy Committee. World Association of Medical Editors. Definition of a Peer Reviewed Journal. Disponible en: http://www.wame.org/resources/ policies\#definition

8 Drummond R. Make peer review scientific: thirty years on from the first congress on peer review, Drummond Rennie reflects on the improvements brought about by research into the process-and calls for more. Nature 2016(535);7610:31-4.

9. COPE Council. Ethical guidelines for peer reviewers. September 2017. www. publicationethics.org

10. Kliewer M, Freed K, DeLong D, et al. Renewing the reviewers: comparison of review quality and reviewer characteristics at the American Journal of Roentgenology. AJR 2005;184:1731-5.

11. Schonhaut L, Cruces P, Cano F, et al.
Vigésimo Encuentro del Grupo de Editores de las Revistas de las Sociedades de Pediatría del Cono Sur. Rev Chil Pediatr. 2016;87:241-4.

12. Spigt M, Arts ICW. How to review a manuscript. J Clin Epidemiol 2010;63(12):1385-90.

13. Prince E. How to write a peer review. Hospital Medicine 2001;62(3):172-5.

14. González de Dios J, González-Muñoz M, Alonso-Arroyo M, Aleixandre-Benavent R. Comunicación científica (XII). Conocimientos básicos para elaborar un artículo científico (7): El sistema de revisión por pares («peer review») a debate: fortalezas y debilidades. Acta Pediatr Esp. 2014;72(5):1038.

15. Cobo E, Cortés J, Ribera JM, et al. Effect of using reporting guidelines during peer review on quality of final manuscripts submitted to a biomedical journal: masked randomised trial. BMJ. 2011;343:d6783. 